\title{
Multi-scale microtomography using synchrotron radiation at beamlines P05/PETRA III and P07/PETRA III
}

Beckmann, Felix, Wilde, Fabian, Hammel, Jörg, Moosmann, Julian, Greving, Imke, et al.

Felix Beckmann, Fabian Wilde, Jörg Hammel, Julian Moosmann, Imke Greving, Lars Lottermoser, Mirko Riedel, Marc Thiry, "Multi-scale microtomography using synchrotron radiation at beamlines P05/PETRA III and P07/PETRA III," Proc. SPIE 11840, Developments in X-Ray Tomography XIII, 118400 U (25 November 2021); doi: 10.1117/12.2596240

SPIE Event: SPIE Optical Engineering + Applications, 2021, San Diego, California, United States 


\title{
Brilliant light for materials science: Industrial applications of synchrotron radiation based microtomography at the tomography instruments by GEMS at PETRA III
}

\author{
Marc Thiry, Julian Moosmann, Jörg U. Hammel, Fabian Wilde, Pidassa Bidola, and Felix Beckmann \\ Helmoltz-Zentrum Hereon/GEMS, Max-Planck-Straße 1, 21502 Geesthacht, Germany
}

\begin{abstract}
GEMS is the user platform of Helmholtz-Zentrum Hereon with a unique infrastructure for complementary research with photons and neutrons. The instruments using synchrotron radiation are operated by Hereon at DESY in Hamburg, the instruments using neutrons are located at the research reactor FRM II of the Heinz Maier-Leibnitz Center (MLZ) in Garching near Munich. GEMS provides methods for applied materials research to user groups from both industry and science. At the PETRA III storage ring of the Deutsches Elektronen-Synchrotron DESY, GEMS operates instruments for tomographic analyses from the micro- to the nanoscale at the beamlines IBL/P05 and HEMS/P07. Photon energies from 10 to $200 \mathrm{keV}$ are routinely used to apply attenuation-contrast and phase contrast microtomography. A robot sample changer enables automated high-throughput measurements. The beamline is therefore of special interest for industrial users, especially from the metal industry sector. In this contribution we introduce the tomography set-up at IBL/HEMS and the possibilities for industrial access, together with examples for tomography of from low absorbing up to challenging highly-absorbing samples.
\end{abstract}

\section{INTRODUCTION}

\subsection{The importance of industrial use at large -scale research facilities}

Industrial use of large-scale research facilities (LSF) such as synchrotrons and neutron sources has become more and more important over the recent years ${ }^{1,2}$. Facilities experience a rise in demand by industrial researchers from various sectors for these unique probes, which are more and more often needed in the process of developing new materials and technologies, especially when laboratory techniques reach their limits. On the other hand, there is an increasing demand by funding bodies to cooperate with industry more closely and increase the percentage of industrial users.

Synchrotron radiation based microtomography $(\mathrm{SR} \mu \mathrm{CT})$ therefore becomes more and more important for industrial $\mathrm{R} \& \mathrm{D}$, which leads to new challenges in efficiency of the measurements and service provided by the beamline staff. Especially for metal processing industry, high energy microtomography can be a vital tool to access information about the internal structures of new alloys and workpieces, e.g. in the field of additive manufacturing, where porosity, inclusions or pores play an important role in properties like stability or duration of the products at real-life conditions ${ }^{3}$.

\subsection{The GEMS platform and the microtomography stations at IBL and HEMS}

The Helmholtz-Zentrum Hereon (former Helmholtz-Zentrum Geesthacht, HZG) operates the GEMS platform, which serves as an infrastructure for complementary research with photons and neutrons. The instruments are located at DESY in Hamburg and at the research reactor FRM II of the Heinz Maier-Leibnitz Center (MLZ) in Garching near Munich. All instruments are mainly designed for engineering materials science related measurements. In this paper we focus on the setup of the microtomography end station operated by Hereon at the High Energy Materials Science Beamline (P07/HEMS) and at the Imaging Beamline (P05/IBL) at the light source PETRA III at DESY ${ }^{4-6}$. The beamline HEMS is optimized for the use of photon energies from $35-200 \mathrm{keV}$ with a bandwidth of $10^{-3}$ (bent Laue monochromator, Si 111) providing a field of view of $6.5 \times 2.5 \mathrm{~mm}^{2}$ (low beta) and $2.5 \times 2.5 \mathrm{~mm}^{2}$ (high beta). At the beamline IBL monochromatic photon energies with a field of view of $6.2 \times 2.5 \mathrm{~mm}^{2}$ are available from 8 to $50 \mathrm{keV}$ with bandwidth of $10^{-4}$ (double crystal Si 111 monochromator) or $10^{-2}$ (double multi-layer monochromator). Both beamlines cover a similar setup for microtomography providing attenuation-contrast and phase-contrast techniques. Furthermore, sample environments can

\footnotetext{
Developments in X-Ray Tomography XIII, edited by Bert Müller 
be shared between both beamlines and by the integration of a sample changing robot high throughput automatized tomographic scans are established. The industrial application makes use of about $10 \%$ of the available beamtime. By the DESY review system $80 \%$ of the beamtime is given to external projects for open research. In recent years the instruments at IBL and DESY became a standard tool for the 3-dimensional characterization in biology, medicine, geology, archeology, and materials science. Novel phase contrast techniques could be established for medical applications. Within in-house project sample environments were integrated and routinely used in external user experiments. ${ }^{7-18}$

\subsection{Access to GEMS facilities for industrial users}

In this context it is important to mention that the access to our instruments for commercial users is organized differently to the access for users from academia. While academic users usually apply for beam time in a peer reviewed process and in case of an approved application the use of the instruments is free, as the results are published in ISI listed journals, proprietary use of the beamlines must be paid for. Hereby the prices for beam time follow a graduated price scale, established by DESY as the organization which operates the storage ring PETRA III. The prices are calculated from the overall operation costs of the facility, broken down to a certain price per hour. Depending on the confidentiality on the data, the prices vary, which means users can get a discount, if they plan to publish their data, but prefer to not participate in the peer reviewed process, due to a need of quicker access or other reasons. Other possibilities for industrial users to gain access to PETRA III beamlines are the cooperation with an academic partner (via the peer reviewed process) or a direct cooperation with GEMS, if the scope fits to our inhouse research topics. In the latter case, there is e.g. the possibility to conduct a co-financed $\mathrm{PhD}$ thesis which covers a certain topic over a longer period of time.

Figure 1 summarizes the various access modes for commercial users.

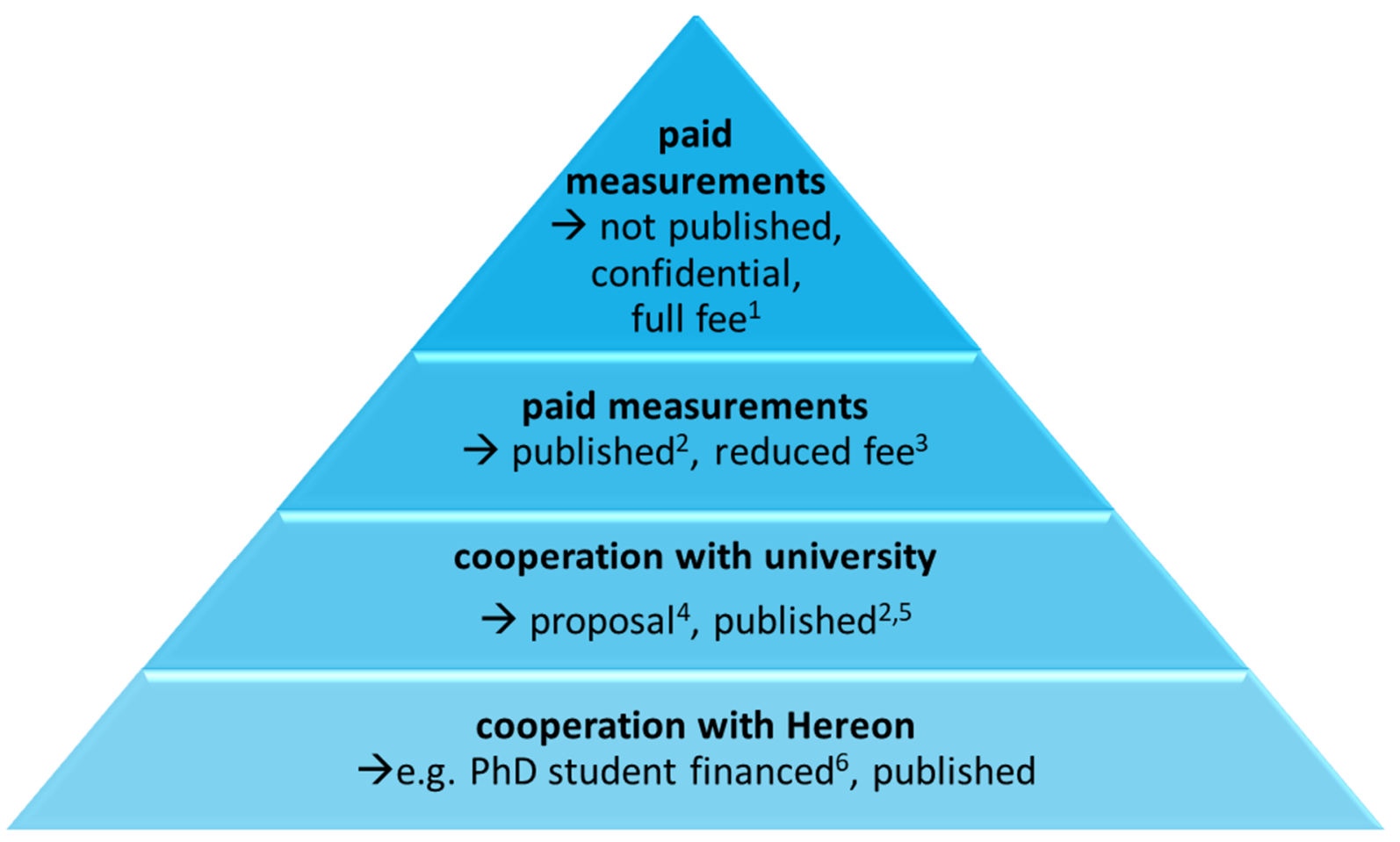

Figure 1: Possibilities and conditions for industry to get access to the synchrotron instruments by GEMS/Hereon. ${ }^{1}$ test possible at zero costs, ${ }^{2}$ in ISI-listed journals, ${ }^{3}$ approx. $25 \%$ reduction, ${ }^{4}$ proposal by academic partner, ${ }^{5}$ critical facts confidential, ${ }^{6}$ contract-based. 


\section{EXAMPLES FOR INDUSTRIAL AND INDUSTRY RELATED MICROTOMOGRAPHY MEASUREMENTS}

On the following pages we will present three examples for industrial or industry related tomography measurements which give a good overview about the capability of the tomography setups at the HEMS beamline and IBL beamline.

Measurement parameters are shown in Table 1.

Table 1 . Tomography parameters

\begin{tabular}{|llll|}
\hline & \multicolumn{2}{c|}{ SAMPLE PARAMETERS } \\
& Circuit board (HEMS) & Aluminum samples (IBL) & AgHf samples (HEMS) \\
\hline Sample size & $4 \times 2 \times 1 \mathrm{~mm}^{3}$ & $4 \mathrm{~mm}$ & $3.8 \mathrm{~mm}$ \\
Photon energy & $80 \mathrm{keV}$ & $30 \mathrm{keV}$ & $175 \mathrm{keV}$ \\
Effective pixel size & $4 \mu \mathrm{m}$ & $4.8 \mu \mathrm{m}$ & $4.2 \mu \mathrm{m}$ \\
\hline
\end{tabular}

\subsection{Circuit board for satellite applications}

Components for the space industry always require the highest quality standards, since faulty components can cause the failure of an entire satellite launch mission. Together with the space system integrator OHB system AG, we investigated use of synchrotron tomography to study the interior of electronic components after manufacturing, integration and different extreme test configurations. The company specialized in satellite production, is interested in potentially using tomography as non-destructive measurement tool on flight relevant hardware including circuit boards. The goal was to visualize the contacts in between board layers, which were produced by specialized methods to match the requirements of applications in space, and to study potential degradations from shock vibration or thermal cycling.

Small circuit board parts of about $4 \times 8 \times 1 \mathrm{~mm}^{3}$ size were scanned with a photon energy of $80 \mathrm{keV}$. Figures 2 and 3 show an exemplary section from the acquired tomogram. 


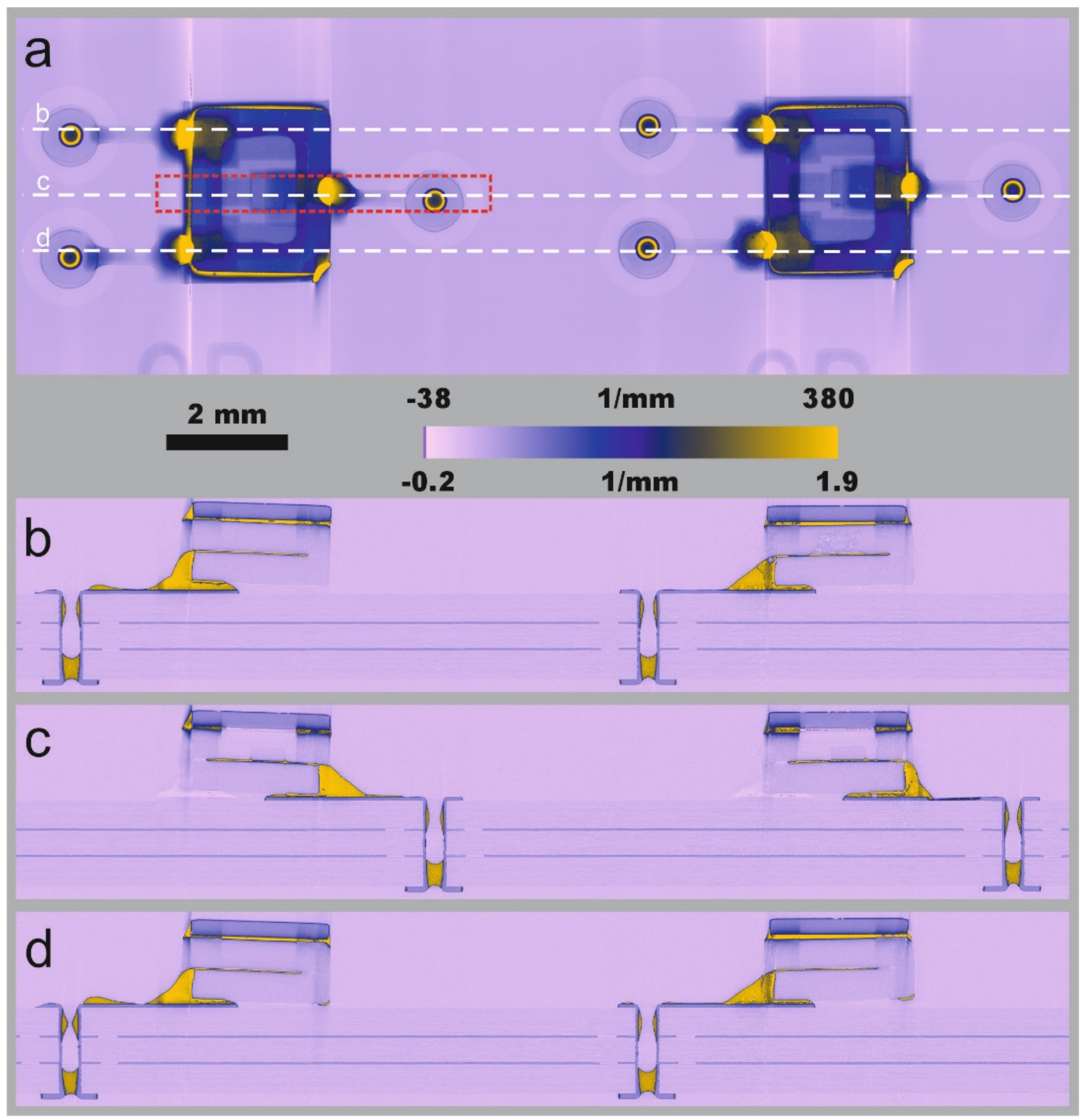

Figure 2: Slices from the tomogram of a circuit board sample; a: top view (total projection of all slices), b, c, d: side views (single slice). The picture shows the components of the circuit board (polymer-fiber composite and various metals or alloys). Of special interest are the brazed parts next to the microchip and in the vias (yellow). While the contacts seem generally intact, some porosity and cracks can be seen in the brazing alloy. The color bar describes the relative attenuation per $\mathrm{mm}$. 


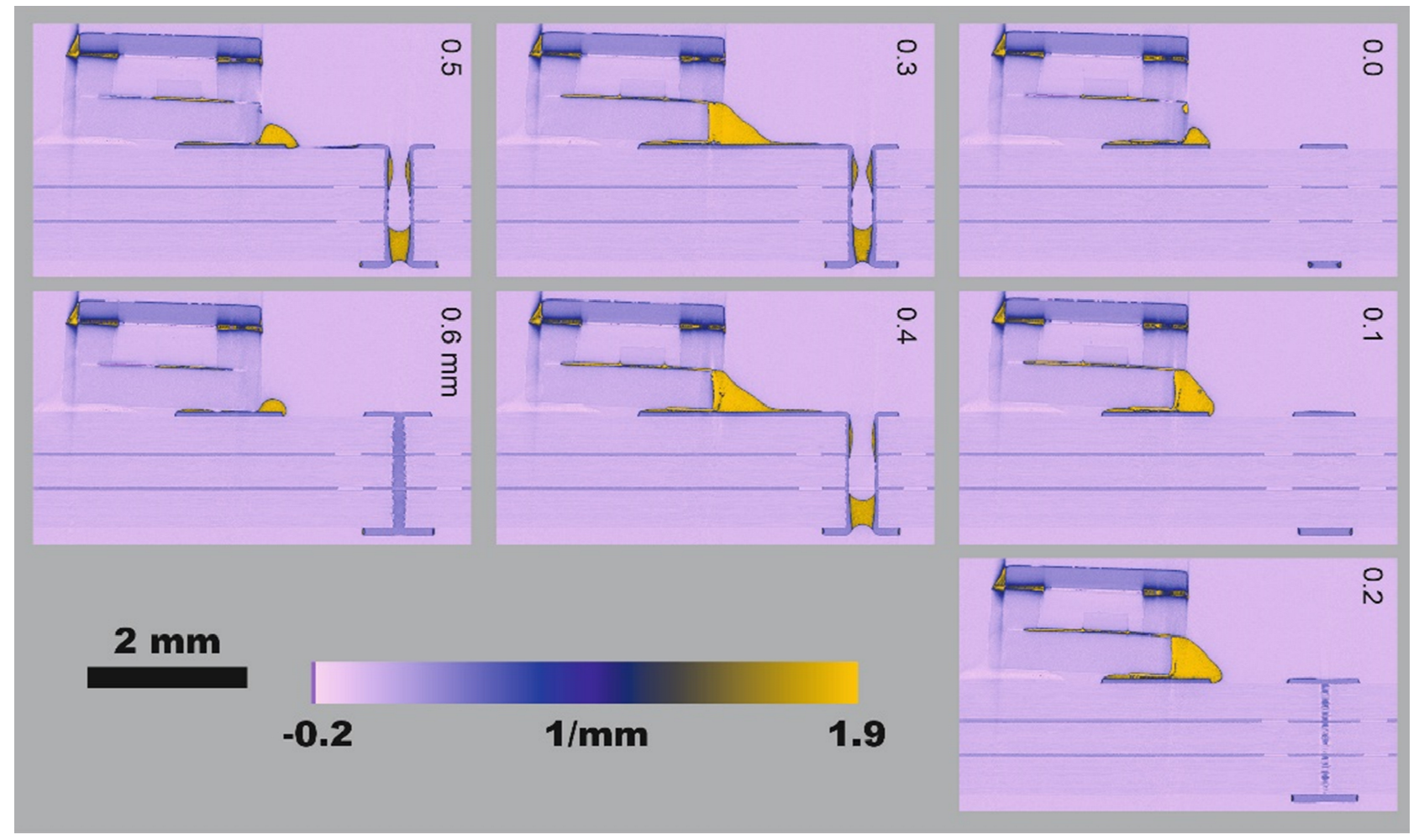

Figure 3: Slices from the circuit board sample tomogram of Figure 2. (red markings). The picture shows the components of the circuit board (polymer-fiber composite and various metals or alloys). Of special interest are the brazed parts next to the microchip and in the vias (yellow). While the contacts seem generally intact, some porosity and cracks can be seen in the brazing alloy. The color bar describes the relative attenuation per $\mathrm{mm}$.

The pictures show that the various components, such as polymer composite of the circuit board and various metals or alloys, can be well distinguished. Of special interest were the brazed parts next to the microchip and in the vias (yellow). While the contacts seem generally intact, some porosity and cracks can be seen in the brazing alloy.

The first results indicated the potential use cases of tomography in quality inspections in space but also in non-space applications. We are considering further systematic studies of similar samples leading to a better lifetime expectation and project risk assessment based on the results.

\subsection{New aluminum alloys for additive manufacturing}

In the framework of the EU Interreg project BalticTRAM, which provided feasibility studies for European companies at large scale research facilities, we received samples of new aluminum-based test alloys from a company working on new powders for additive manufacturing. Various alloys were used to create test samples by selective laser melting. Tomography at the beamline IBL/P05 was used to determine cracks and pores in the test samples, which helped to determine the quality of SLM samples produced by variation of the SLM parameters. Figure 4 shows an example of one of the scanned specimens. It shows porosity as well as cracks in the material. The cracks are highlighted here by a color spectrum and form an adjacent network through the whole sample. 


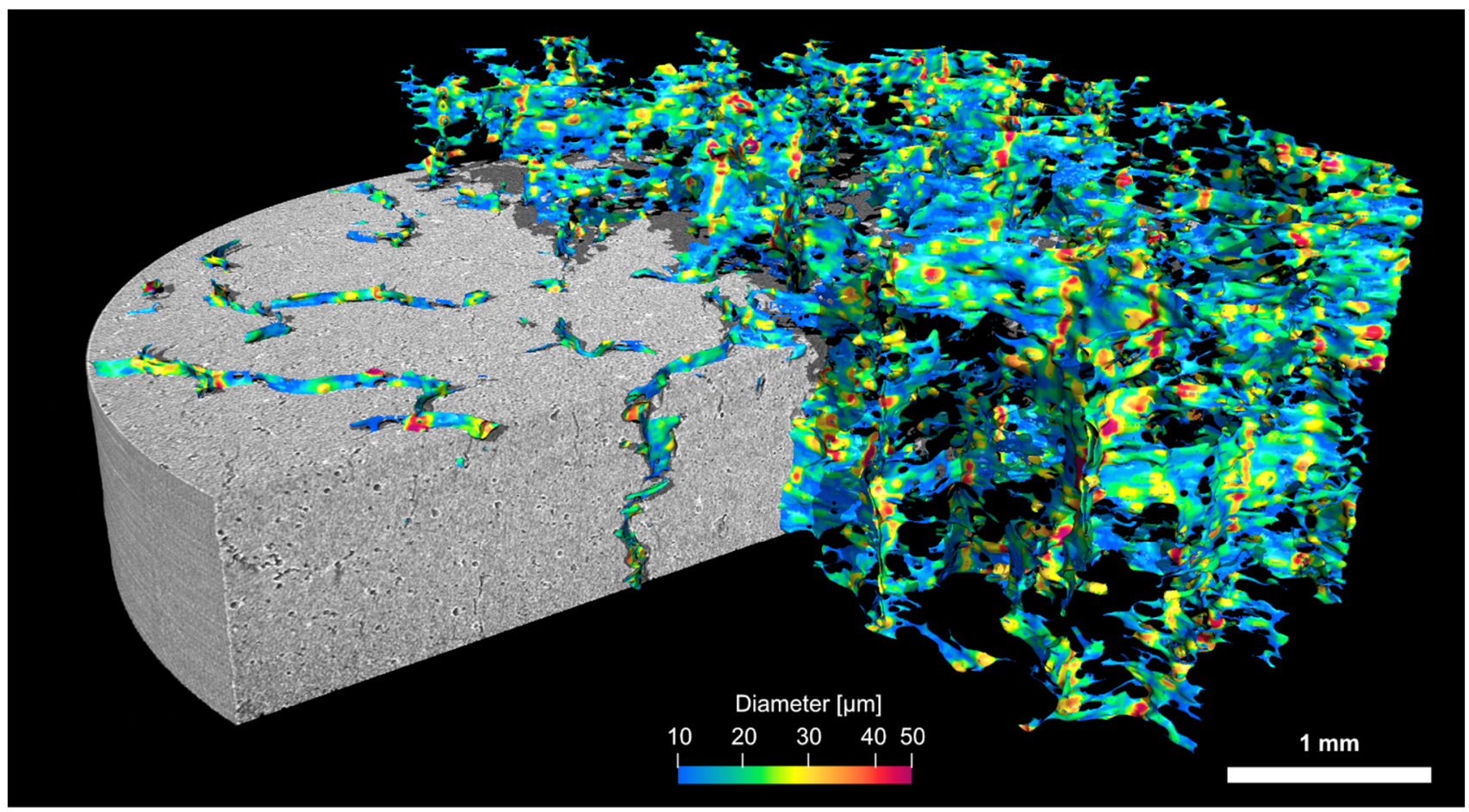

Figure 4. Visualization of cracks in a SLM generated aluminum alloy sample. The highlighted cracks form an adjacent network. Crack diameters are visualized by color variation as explained in the legend. The solid components are shown in gray scale, partially clipped away for better visibility of the network of cracks.

\subsection{Highly absorbing hafnium component}
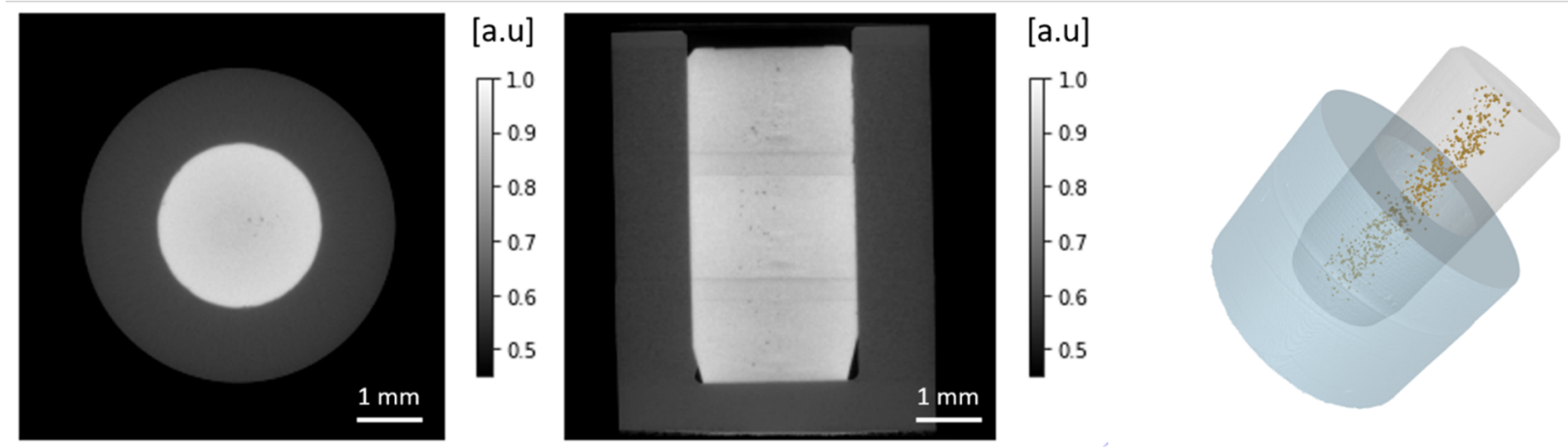

Figure 5. Tomogram of AgHf-sample. The high energy x-rays at HEMS/P07 enable an insight in this highly absorbing sample and make pores in the hafnium core as well as voids between the two components visible.

One of the most challenging industrial requests for the HEMS microtomography has been recently provided by Carl Zeiss QEC GmbH, Germany. For one of their customers, they needed tomography of a highly absorbing sample made of silver with a hafnium core. Since this is even with longest acquisition times not feasible with laboratory-based x-ray tomography, a high energy synchrotron beamline became an obvious choice. Figure 5 shows both the porosity of the hafnium core and the voids between hafnium core and silver shell. 


\section{CONCLUSION}

This article shows, that synchrotron based microtomography, besides its high value for academic research, also offers great opportunities for industry relevant topics. Here we focused on mainly metallic samples, from low absorbing aluminum to highly absorbing hafnium components, which showcases the broad spectrum of materials which can be analyzed by the GEMS instruments, be it the mid-energy range at IBL/P05 or the high-energy regime at HEMS/P07.

Access to the facilities is possible either by cooperation with academic partners and the peer reviewed application process or by proprietary access for which exists a variable pricing model, enabling cost reduction if results are (partially) published.

\section{ACKNOWLEDGEMENTS}

We would like to thank our industrial partners for providing the samples and agreeing to publish the results in this article: Circuit board: Axel Müller at OHB System AG; AgHf-Sample: Dr. Udo E. Frank, ZEISS Group. The partner who provided the SLM-generated Aluminum samples needs to remain confidential. Instead, we thank the EU Interreg project \#R002 Baltic TRAM-funding of the measurements. The industrial imaging has been performed within the DESY beamtime IDs c20181120_000_btram (Al-sample, IBL), ID 11007655 (circuit board, HEMS), ID 110101206 (AgHf-sample, HEMS).

\section{REFERENCES}

[1] Lawniczak-Jablonska, K. and Cutler, J., "X-ray techniques for innovation in industry”, UCrJ 1(6), 604-613 (2014)

[2] Hormes, J. and Warner J., Industrial Accelerators and Their Applications, pp. 371-412 (2012)

[3] Kojola, N., Lindberg, F., Larsson, E “Tomography for clean steel” Vinnova, April 2020, https://www.vinnova.se/en/p/tomography-for-clean-steel/

[4] F. Beckmann, J. U. Hammel, J. Moosmann et al., "Optimization of high-energy microtomography using synchrotron radiation at PETRA III," Proceedings of SPIE - Developments in X-Ray Tomography XII, 11113, (2019).

[5] F. Wilde, M. Ogurreck, I. Greving et al., "Micro-CT at the imaging beamline P05 at PETRA III," AIP Conference Proceedings, 1741(1), 030035 (2016).

[6] I. Greving, F. Wilde, M. Ogurreck et al., [P05 imaging beamline at PETRA III: first results], San Diego, California, United States (2014).

[7] M. Riedel, A. Gustschin, L. Ushakov et al., "High-resolution quantitative phase-contrast x-ray imaging for biomedical samples at PETRA III," Proceedings of SPIE - Developments in X-Ray Tomography XIII, 11840, (2021).

[8] D. Zander, P. Zaslansky, N. A. Zumdick et al., "The Effect of Chemistry and 3D Microstructural Architecture on Corrosion of Biodegradable Mg-Ca-Zn Alloys," Advanced Engineering Materials, n/a(n/a), 2100157 (2021).

[9] M. Wallmeier, C. Barbier, F. Beckmann et al., "Phenomenological analysis of constrained in-plane compression of paperboard using micro-computed tomography Imaging," Nordic Pulp \& Paper Research Journal, (2021).

[10]D. Tolnai, S. Gavras, F. Wilde et al., "In Situ Synchrotron Tomography of the Solidification of an Electron 21 Mg Alloy," Advanced Engineering Materials, n/a(n/a), 2100383 (2021).

[11]F. Tang, Z. Wu, C. Yang et al., "Synchrotron X-Ray Tomography for Rechargeable Battery Research: Fundamentals, Setups and Applications," Small Methods, n/a(n/a), 2100557 (2021).

[12]F. Sun, L. Duchêne, M. Osenberg et al., "Na electrodeposits: a new decaying mechanism for all-solid-state Na batteries revealed by synchrotron X-ray tomography," Nano Energy, 82, 105762 (2021).

[13] S. Schmelzle, S. Bruns, F. Beckmann et al., "Using In Situ Synchrotron-Radiation-Based Microtomography to Investigate 3D Structure-Dependent Material Properties of Tension Wood," Advanced Engineering Materials, 2100235 (2021).

[14] J. Sartori, S. Köhring, S. Bruns et al., "Gaining Insight into the Deformation of Achilles Tendon Entheses in Mice," Advanced Engineering Materials, n/a(n/a), (2021).

[15] M. Neumann, E. Machado Charry, E. Baikova et al., "Capturing Centimeter-Scale Local Variations in Paper Pore Space via $\mu$-CT: A Benchmark Study Using Calendered Paper," Microscopy and Microanalysis, 1-11 (2021). 
[16] T. E. Koch, D. Spahr, B. J. Tkalcec et al., "Formation of chondrule analogs aboard the International Space Station," Meteoritics \& Planetary Science, n/a(n/a), (2021).

[17] E. Euchler, R. Bernhardt, F. Wilde et al., "First-Time Investigations on Cavitation in Rubber Parts Subjected to Constrained Tension Using In Situ Synchrotron X-Ray Microtomography (SR $\mu \mathrm{CT})$,” Advanced Engineering Materials, 2001347 (2021).

[18] A. Gapeeva, J. Vogtmann, B. Zeller-Plumhoff, et al., "Electrochemical Surface Structuring for Strong SMA Wire-Polymer Interface Adhesion", ACS applied materials \& interfaces 13 (18), 21924-21935 (2021). 